\title{
Evaluation of Mesh-Enhanced VANET Deployment Models
}

\author{
Niranjan Potnis ${ }^{a}$, Atulya Mahajan $^{a}$, Kartik Gopalan $^{b}$, Andy Wang $^{a}$ \\ ${ }^{a}$ Computer Science, Florida State University \\ ${ }^{b}$ Computer Science, State University of New York at Binghamton \\ Contact:kartik@cs.binghamton.edu, awang@cs.fsu.edu
}

\begin{abstract}
While wireless vehicular ad hoc networks (VANETs) are attracting greater commercial interest, current research has not adequately captured the real-world urban constraints in VANET deployment. In this work, we evaluated the feasibility and benefits of deploying a VANET in urban settings with a wireless mesh backbone infrastructure. We modeled urban street layouts, traffic rules, RF attenuation due to physical obstacles, and the use of multiple radio channels Our results indicate that the performance improves in dense networks when routing decisions are limited to mesh nodes, whereas it improves in sparse networks when mobile nodes also participate in routing. We also show that the effect of signal attenuation due to physical obstacles can potentially be parametrized in simulations using empirical real-world measurements.
\end{abstract}

\section{INTRODUCTION}

Vehicular ad hoc networks (VANETs) have generated increasing research and commercial interest due to promising applications. Mobile communication, traffic monitoring, safety, and public utility management are some examples. One model for VANET deployment is a pure vehicle-tovehicle (V2V) wireless communication network with no static wireless infrastructure support. In a mobile environment, this model can experience a high degree of churn in network routes and hence frequent breakages. To stabilize network routes, an alternative VANET deployment model is through a wireless mesh network which supports or auguments vehicular connectivity. To date, wireless mesh networks have been primarily treated as a means to provide broadband Internet access to residential subscribers. This paper evaluates the benefits of using a wireless mesh network as a backbone support infrastructure for VANETs, specifically by deploying the mesh nodes at street intersections. This deployment model combines two recent wireless technologies - wireless mesh networks and ad hoc wireless network between vehicles and static nodes. Consequently, the practicality and performance characterizations of this deployment are not well understood.

We investigated two VANET deployment scenarios: (a) Both vehicles and mesh nodes participate in an ad hoc routing protocol. (b) The routing is limited to the mesh nodes and vehicles are simply communication endpoints. We also explored the performance implications for factors such as traffic rules, street layouts, the use of multiple radio channels, and radio signal attenuation due to physical obstacles. The movement pattern of vehicles, or the mobility model, is a key component of VANET simulations. Traditional mobility models tend to ig- nore urban constraints on vehicular movements. Consequently, the simulation results are unlikely to reflect the real-world performance. For example, the widely used Random Waypoint Model (RWM) [1] assumes that nodes move in an open field without obstructions. In contrast, the layout of roads, intersections with traffic signals, buildings, and other obstacles in urban settings constrain vehicular movement. In our earlier work [2], we introduced three mobility models that capture vehicular mobility vehicular mobility patterns at various levels of detail. In this work, we use the Traffic Light Model (TLM), the most detailed among the three models. The TLM enables us to constrain the movement of mobile nodes according to urban street layouts, speed limits, coordinated traffic signals, and vehicular acceleration and deceleration.

Our major research results can be summarized as follows. Network performance improves in dense networks when routing decisions are limited to mesh nodes, whereas the performance improves in sparse networks when mobile nodes also participate by exploiting opportunistic local connectivity. Using multiple radio channels for communication among mesh nodes, even when using simple and static channel assignment, leads to significant performance improvements due to reduction in channel contention. Finally, the effect of physical obstacles on wireless signal attenuation can potentially be parametrized in simulations based upon empirical real-world measurements.

\section{Mesh-Enhanced Deployment ScEnarios}

We considered the VANET deployment in an urban setting in which a wireless multi-hop network of stationary mesh nodes enables or supplements the network connectivity among mobile nodes. Mesh nodes can be strategically positioned at a subset of street intersections. We evaluated two deployment scenarios: (1) mesh-enhanced peer-to-peer routing (MEPPR) where both the mobile nodes and static mesh nodes participate in routing, and (2) mesh-enhanced infrastructural routing (MEIR) where only the static mesh nodes participate in routing and forwarding packets generated by mobile nodes.

Implementing the two deployment models above involved enhancing the NS2 simulator. First, the TLM mobility pattern generator was altered to designate a subset of nodes as mesh nodes that are positioned at street intersections and remain stationary throughout the simulation. The identity of the mesh nodes and their positions are specified from a separate file. 
The implementation of peer-to-peer routing reuses AODV's implementation in NS2, which allows every node to participate in routing decisions. However, the MEIR deployment required changes to the AODV implementation to ensure that only the mesh nodes participate in routing and forwarding, whereas mobile nodes act as sources and destinations.

\section{The Effect Of Obstacles}

Besides confining the vehicle movements to streets, physical obstacles also affect radio signal propagation through attenuation, reflection, diffraction, and refraction. Traditionally, pure analytical models have limitations in capturing complex real-world factors that influence radio signal strengths. Our approach is to use empirically measured data from real-world urban settings to characterize the impact of different factors on radio signals in a few simulation parameters. We measured the signal strength variation from a commodity access point around two city blocks in downtown Tallahassee - including a $100 \mathrm{~m} \times 100 \mathrm{~m}$ block with several three-story buildings and a $200 \mathrm{~m} \times 50 \mathrm{~m}$ block with one-story buildings. We placed an 802.11b Linksys wireless access point at a corner of the block being measured. We then used the Wavemon [3] tool running on a Linux laptop equipped with a wireless PCI card to take signal strength measurements at various locations around the block. The empirical data were composed of the distances from the access point and the associated signal strength.

A logarithmic transformation was performed on collected distances before a linear regression was applied on the signal strength, $S$ in decibels/milliwatts $(\mathrm{dBm})$, as a function of distances, $d$ in meters [4]. Logarithmic linear regressions yielded the following formulas, with $R^{2}$ (coefficient of determination) of 0.6836 and 0.9698 , indicating that $68 \%$ and $97 \%$ of the variances in data are explained by these equations respectively.

$$
\begin{aligned}
& \text { Block } 1: S=-25.809-29.773 * \log (d) \\
& \text { Block } 2: S=-20.089-33.012 * \log (d)
\end{aligned}
$$

From the structure of Equations 1 and 2, we can derive a (simplified) parametrization of the received signal strength.

$$
P_{r}=P_{t}+A-B \log (d)
$$

$P_{r}$ and $P_{t}$ are the signal strengths (in $\mathrm{dBm}$ ) at the receiver and the sender respectively; $d$ is the distance between the two in meters, and $A$ and $B$ are tunable parameters whose significance we will explore below.

Interestingly, the radio propagation models used in NS2 can also be represented in the form of Equation 3. The two principal radio propagation models used in NS2 are the Friis model for free space propagation and the two-ray ground model that accounts for multipath reflection from the ground.

$$
\begin{gathered}
\text { Friis : } P_{r}(d)=\frac{P_{t} G_{t} G_{r} \lambda^{2}}{(4 \pi)^{2} d^{2} L} \\
\text { Two ray ground: } P_{r}(d)=\frac{P_{t} G_{t} G_{r} h_{t}^{2} h_{r}^{2}}{d^{4} L}
\end{gathered}
$$

$P_{r}(d)$ is the received power (watts) at distance $d, P_{t}$ is the transmit power (watts), $G_{t}$ and $G_{r}$ are antenna gains

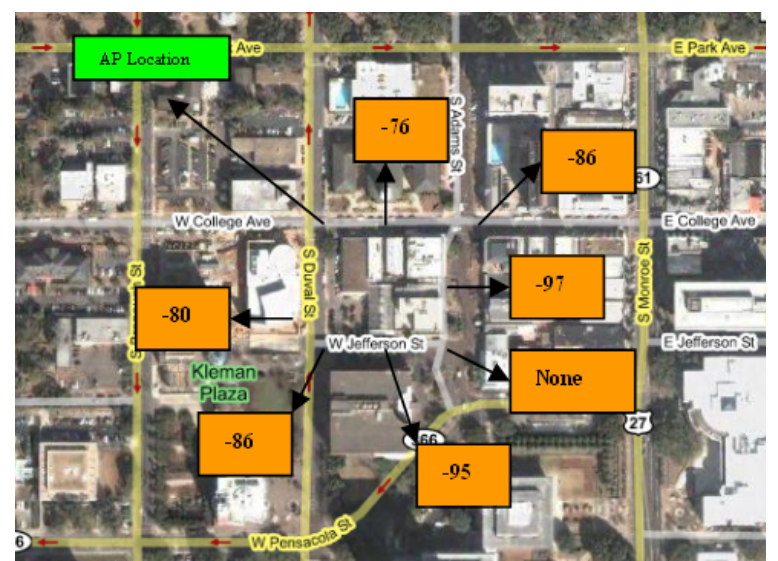

Fig. 1. Transmission signal strengths measured around a block in downtown Tallahassee. AP is the access point location while the numbers indicate signal strength in $\mathrm{dBm}$.

for the transmitter and the receiver respectively, $h_{t}$ and $h_{r}$ are the antenna heights for the transmitter and the receiver respectively, $L$ is the system loss, and $\lambda$ is the wavelength. Both Equations 4 and 5 could be represented in the form of Equation 3, after converting watts into $\mathrm{dBm}$. The conversion is given by $P(d B m)=10 \log P(w)+30$. From the Friis mode in Equation 4

$$
\begin{aligned}
& P_{r}(d B m)=10 \log \left(\frac{P_{t} G_{t} G_{r} \lambda^{2}}{(4 \pi)^{2} d^{2} L}\right)+30 \\
& =10 \log \left(P_{t}\right)+30+10 \log \left(\frac{\alpha}{d^{2}}\right), \text { where } \alpha=\frac{G_{t} G_{r} \lambda^{2}}{(4 \pi)^{2} L} \\
& =P_{t}(d B m)+10 \log \alpha-20 \log (d) \\
& =P_{t}(d B m)+A-B \log (d)
\end{aligned}
$$

Similar derivation can be performed for the two-ray ground equation. The default values of $A$ and $B$ in NS2 for the Friis model are $A=-31$ and $B=20$; for the two-ray ground model, $A=7.5$ and $B=40$.

If we assume that the received signal strength depends only on the presence of obstacles and the distance from the sender, then $A$ and $B$ can be interpreted as follows. Parameter $A$ captures the constant factor reduction in signal strength due to the presence of obstacles in a particular terrain. Parameter $B$ captures the order of magnitude reduction in the signal strength with the distance from sender, the order of magnitude being determined by nature of the obstacles. We will refer to $A$ as the constant factor and $B$ as the distance factor in the remainder of the paper.

We modified the radio propagation model of NS2 according to Equation 3, such that parameters $A$ and $B$ can be specified as configuration parameters to the simulation. Of course, the actual values of $A$ and $B$ would be quite different for various urban settings, and even across different regions within a single urban setting. Regardless, these two parameters give us convenient knobs to capture and explore the effect of obstacles in VANET simulations.

\section{Multi-Radio Deployment}

Wireless channel contention can be mitigated through the use of multiple radio channels, which enables each static mesh node to communicate over multiple wireless channels, 
via possibly different network interfaces. We used the NS2 extensions proposed in [5], which allow wireless nodes to be configured with multiple network interfaces, each operating on a different radio channel. There are 11 radio channels (1-11) specified in the $802.11 \mathrm{~b} / \mathrm{g} 2.4 \mathrm{GHz}$ spectrum, with adjacent channels overlapping on a $5 \mathrm{MHz}$ band. Effective use of multiple radios in a mesh network requires assigning channel numbers to each interface such that (1) network connectivity is maintained, i.e., any two communicating neighbors share at least one channel, and (2) channel contention among neighboring nodes is minimized. We implemented two channel assignment approaches: static and dynamic. In both approaches, all mesh nodes have the same number of network interfaces with each interface assigned to a fixed subset of channel numbers. In the static approach, every mesh node equally divides all of its mesh neighbors among the selected channels for communication. For example, a mesh node with three network interfaces and six neighbors could transmit packets to two neighbors on each of the non-overlapping channels 1 , 6 , and 11 using one interface per channel. In the dynamic channel assignment approach, each node tracks the network load observed on each channel over a recent time window. A node transmits an outgoing packet over the least loaded channel. Thus the channel used for communication between any two neighbors can vary over time with network load. We implemented both assignment approaches by enhancing the multi-radio extensions to NS2 [5] and also altered the AODV implementation such that packet transmissions to neighbors occur only on the channel assigned to that interface.

\section{EVALuATions}

In this section, we investigate the performance of various mesh-enhanced VANET deployment scenarios. The simulations were performed using the AODV protocol in NS2 [6] over a grid topology of a $1200 \mathrm{~m} \mathrm{X} 1200 \mathrm{~m}$ area with $200 \mathrm{~m}$ X 50m city blocks and 171 street intersections. Static mesh nodes were positioned at a subset of street intersections randomly chosen using the uniform distribution. Mobile nodes follow mobility pattern generated by TLM. All nodes have a transmission range of $250 \mathrm{~m}$. Each simulation run lasts for 1350s, including 450s initial warm up time. Each data point is averaged over 10 runs with different initial placements of mesh nodes and mobility patterns.

\section{A. Mesh-Enhanced Peer-to-Peer vs. Infrastructural Routing}

In this section, we try to understand the performance of MEPPR and MEIR without considering multiple radios or obstacles. Figure 2 shows the effects of varying the number of mobile nodes on the delivery ratios in the MEPPR deployment scenario. The two plots correspond to fixed number of mesh nodes at 171 mesh nodes (one per intersection) and 40 (approximately 23\% of intersections). For 171 mesh nodes, as the number of nodes participating in the routing process increases and the network becomes dense, the resulting channel contention increases. Consequently, the delivery ratio of MEPPR deployment degrades. On the other hand, with only 40 mesh nodes, the MEPPR deployment maintains a high delivery ratio with the addition of mobile nodes. To rule out the performance degradation due to only the total number of mobile and static nodes, we also extended the number of mobile nodes to 170 (not shown in the plot) and observed no significant performance degradation with only 40 mesh nodes. These results confirm our hypothesis that the channel access contention generated by the number of nodes participating in the routing process is an important factor in VANET's performance.

Figure 3 shows the effects of increasing the number of mobile nodes on the delivery ratios under the MEIR deployment. We again used 171 and 40 mesh nodes. The plot shows that the delivery ratios do not vary significantly for either setting as the number of mobile nodes increases. Since the mobile nodes do not participate in the routing process (they are merely sources and sinks for data packets), and the mesh nodes that participate in routing decisions are stationary, the resulting routes are much more stable than in MEPPR. MEIR routes change only when the mobile endpoints move out of range of their immediate next hop mesh node.

Interestingly, in the sparser case of 40 mesh nodes in MEIR, since the mobile nodes do not participate in the routing process, the resulting network coverage and connectivity is poorer than the case of 171 MEIR mesh nodes (in Figure 3 and 40 MEPPR mesh nodes (in Figure 2), Consequently, the delivery ratio is also lower (around 90\%).

Nest, we fixed the number of mobile nodes to either 30 or 35 and varied the number of stationary mesh nodes. As shown in Figure 4, since both mesh nodes and mobile nodes perform routing, a relatively small number of mobile nodes combined with mesh nodes can achieve good routing coverage and delivery ratio. On the other hand, too many mesh nodes severely limit the number of mobile nodes due to channel contention. This is seen in the case of 35 mobile nodes and 171 mesh nodes in the above graphs. Figure 5 shows how the number of mesh nodes affects the delivery ratios when only the mesh nodes perform routing. Clearly, a sufficient number of mesh nodes are needed to achieve good routing coverage and delivery ratio. However, this deployment model scales better when compared to MEPPR because the communication paths among mobile nodes are more static, resulting in fewer route breakages and fewer route discovery and recovery events. Figure 5 indicates that the delivery ratios across all numbers of mesh nodes are not as sensitive to the number of mobile nodes.

Figure 6 shows the effects of varying the number of constant bit rate (CBR) sources on delivery ratios. Here, both the number of mobile nodes and the number of mesh nodes are fixed at 80 . By increasing the number of CBR sources, network traffic is increased. The performance degrades in both deployment scenarios as the number of CBR sources increases. However, the MEIR deployment scenario consistently outperforms the MEPPR deployment, demonstrating how static routing nodes can scale better compared to a mixture of static and mobile routing nodes. Routing with mobile nodes 
significantly increases the number of route breakages and resulting control traffic for recovery. On the other hand, static routing nodes limit route breakages to the mobile end points.

Results from this subsection suggest that in a dense network, where the total number of nodes is high, MEPPR deployment can lead to decreased performance as a result of channel contention. In addition, MEIR deployment can scale better with increased network loads. On the other hand, in a sparse network with a smaller number of nodes, MEPPR deployment provides better routing coverage and higher connectivity.

\section{B. Multi Radio Deployment}

Figure 7 shows how the number of mobile nodes affects the performance of single-radio and multi-radio MEPPR deployments, With two channels, even MEPPR can deliver constant network performance, confirming that the decrease in performance for single-radio deployment in Figure 2. By providing an additional channel, channel contention is significantly reduced.

Figure 8 shows how the number of CBR sources affects network performance for both channel and routing configurations. With two channels, both routing configurations perform better compared to their single-radio counterparts. This is because the network capacity to carry traffic is almost doubled. The performance of MEPPR does not exhibit channel contention until a high number of CBR sources are employed. MEIR with two channels results in a very high capacity network that can handle high network loads.

Figure 9 shows how the number of channels affects network performance for the MEPPR deployment scenario. As the number of channels increases, the delivery ratio peaks at three channels, followed by degraded performance for four and five channels. Intuitively, more channels available for communication should increase network capacity and hence performance. However, according to the 802.11 standard, among the 11 channels, communicating through a single channel can interfere with the two channel numbers above and below. The maximum number of channels that can be assigned for concurrent transmission without interference is three (1, 6, and 11). Beyond that, it is no longer possible to use more channels without interfering with adjacent channels. Alternatively, performance degrades when the number of channels exceeds three. It is interesting to note that the performance with four channels is lower as compared to that with a single channel, primarily due to inter-channel interference. The $802.11 \mathrm{RTS} / \mathrm{CTS}$ mechanism is used within a single channel and not across multiple channels. Hence, in case of four channels, even though a channel is clear to send, it may not be free of interference from other channels.

Figure 10 demonstrates how the number of CBR sources affects network performance with the dynamic and static channel assignment methods described in Section IV. The experiment includes two channels and uses MEPPR deployment. In the static approach, every node distributes its routes to neighbors equally among its network interfaces. The channel number associated with a particular interface is fixed over time. In the dynamic channel assignment approach, the interface, and hence the channel number chosen for communication with a neighbor may vary at packet arrival times. The dynamic approach has an edge over the static one as any available channel can be chosen for communication. This advantage can be seen with high numbers of CBR sources.

\section{Obstacle Representation}

Figure 11 shows how obstacle factor $\mathrm{A}$ in Equation 3 affects network performance for both routing configurations. An increasingly negative value of obstacle factor A should lead to a decrease in signal strength at receivers and decrease performance. This is observed when $\mathrm{A}<-35$ for both routing configurations. The default value of A in the NS2 propagation model is -31 , which corresponds to a total absence of obstacles. However, it is interesting to note that in the MEPPR deployment scenarios, when $\mathrm{A}>-15$, performance degrades. For such a decreased negative value of obstacle factor A, the signal strength at the receiver is high enough to cause unwanted reception and interference among these receptions. This is not observed for MEIR because because the static mesh nodes maintain a fixed distance from one another throughout the simulations.

Figure 13 shows how the distance factor B in Equation 3 affects network performance for both mesh-enhanced routing configurations. A more positive value of distance factor B should reduce signal strength at receivers and decrease performance. This is observed in cases of values of B $>21$ for both routing configurations. However, MEPPR deployment performs better as compared to MEIR deployment. This is the result of the mobile nodes' participation in routing to enhance connectivity and coverage. For a high value of distance factor, network connectivity is still better in the MEPPR deployment as more nodes are reachable through the mobile nodes.

\section{RELATED WORK}

To date, studies in the fields of mesh networks, obstacle modeling in wireless communication, mobility modeling, and multi-radio networks have been largely performed in isolation. To the best of our knowledge, this paper is the first work to synthesize and systematically evaluate the impact of these factors on urban VANET deployment. Many vehicular mobility models have been developed to study VANET performance. [7] and [8] proposed mobility models for the urban environment. [2] proposed mobility models to capture different levels of mobility details and noted the effects and importance of vehicular movement on network performance. Several deployment scenarios were presented are possible for connecting in-vehicle systems: a pure wireless V2V architecture, a V2V architecture with a wired backbone and wireless last hops, and hybrid architectures with a combination of $\mathrm{V} 2 \mathrm{~V}$ and a wired infrastructure. [9] presents a mobility-centric approach for data dissemination in vehicular networks. [10] proposed MCTP, a TCP-based transport protocol for Internet access in vehicular environments. [11] presents a new scheme 


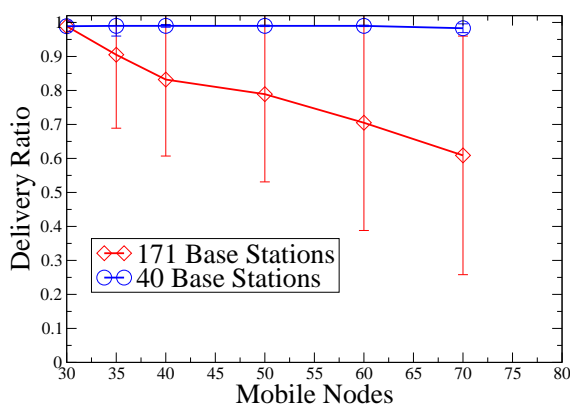

Fig. 2. Variation of delivery ratio with number of mobile nodes in MEPPR deployment.

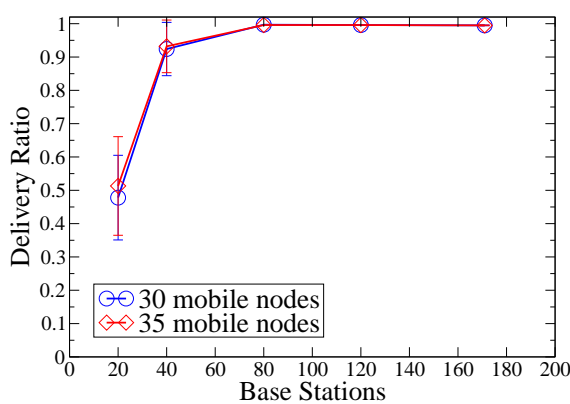

Fig. 5. Variation of delivery ratio with number of mesh nodes in MEIR deployment.

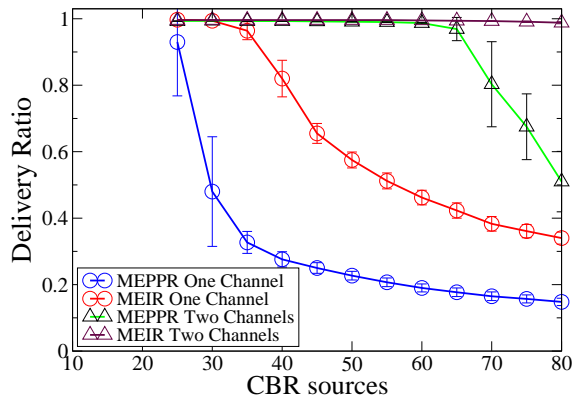

Fig. 8. Variation of delivery ratio with number of CBR sources for both MEPPR and MEIR with 171 mesh nodes and either one or two channels.

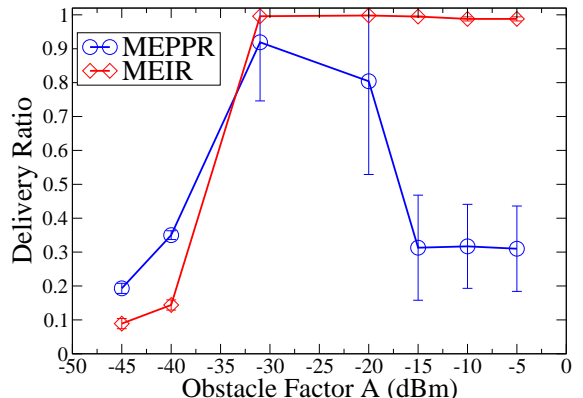

Fig. 11. Variation of delivery ratio with obstacle factor A in Equation 3 for both MEIR and MEPPR deployments with 171 mesh nodes and 35 mobile nodes.

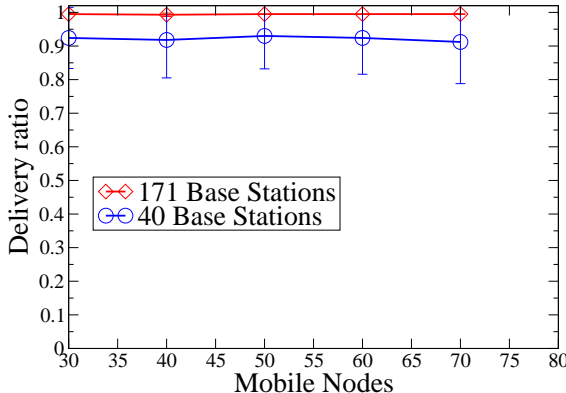

Fig. 3. Variation of delivery ratio with number of mobile nodes in MEIR deployment.

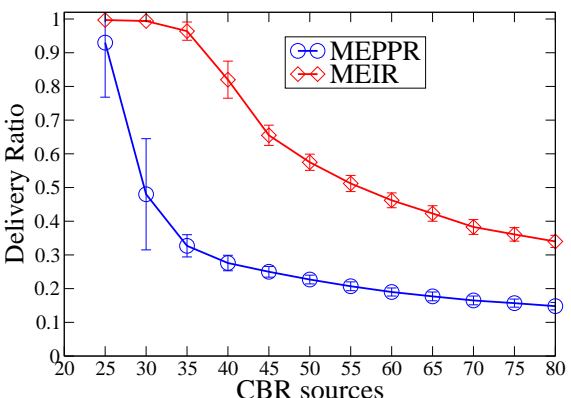

Fig. 6. Variation of delivery ratio with number of CBR sources for both MEPPR and MEIR deployment.

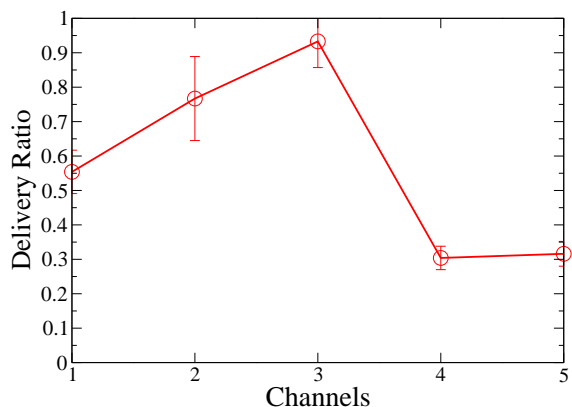

Fig. 9. Variation of delivery ratio with number of channels for MEPPR deployment with 171 mesh nodes.

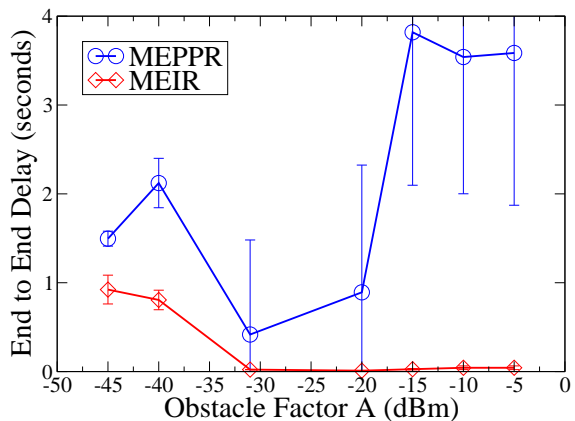

Fig. 12. Variation of end to end delay with obstacle factor $\mathrm{A}$ in Equation 3 for both MEIR and MEPPR deployments.

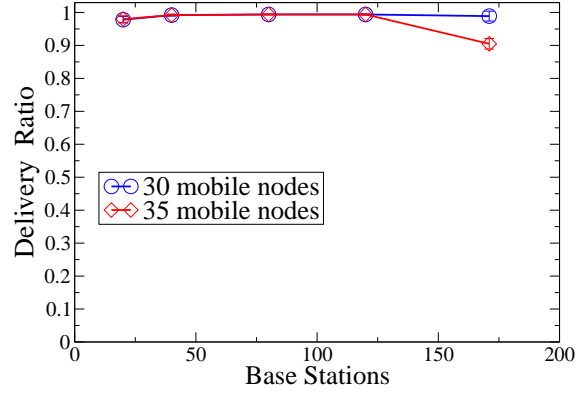

Fig. 4. Variation of delivery ratio with number of mesh nodes in MEPPR deployment.

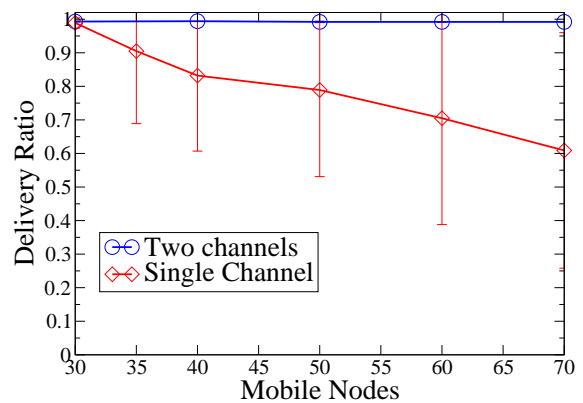

Fig. 7. Variation of delivery ratio with number of mobile nodes for MEPPR with 171 mesh nodes and and either one or two channels.

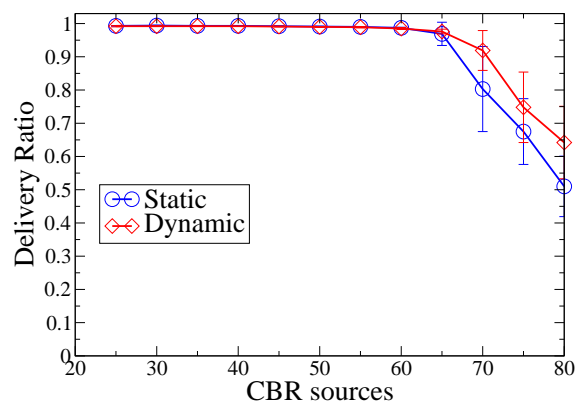

Fig. 10. Variation of delivery ratio with static and dynamic channel assignment approaches in MEPPR deployment with 171 mesh nodes.

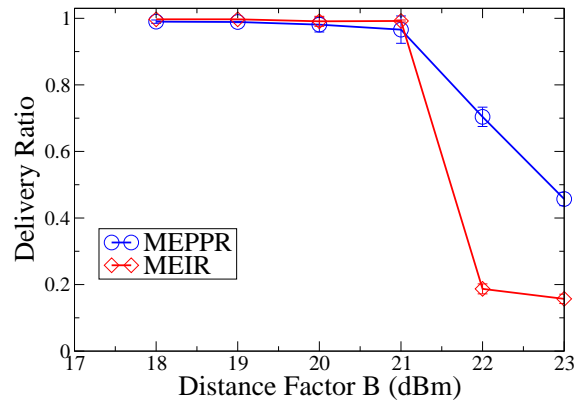

Fig. 13. Variation of delivery ratio with distance factor B in Equation 3 for both MEIR and MEPPR deployments with 171 mesh nodes and 35 mobile nodes. 
called Directional Propagation Protocol for information warning functions in vehicular networks. The FleetNet Project [12] investigated multi-hop inter-vehicular communication, where vehicles form separate clouds of ad hoc networks and roadside installed gateways provide Internet service to vehicles. The focus is on protocol interoperability for Internet integration into vehicular systems. [13] studied characteristics of vehicular mesh networks and invented routing algorithms for commercial applications such as distributed sensing and computing. The work considered vehicles as routers that interconnect with end-point gateways to form vehicular mesh networks. In mesh network Microsoft [14] has proposed deployment of self-organizing community mesh networks. The RoofNet project [15] provides residential broadband Internet access. Motorola has proposed mesh network solutions for intelligent transport systems and vehicular communication [16]. However, most efforts are devoted to setting up and enhancing mesh networks for Internet access, as opposed to exploring ways to support VANETs. [17] showed that with an efficient channel assignment of two channels per node, the network performance can improve by a factor of 8 over single-channel networks. Our work confirms confirms this observation for mesh-enhanced VANETs. The effect of obstacles on wireless networks is relatively little studied. [18] points out that commonly used radio propagation models for indoor MANET evaluations are highly inaccurate and relative protocol performance varies highly depending upon the model. [19] models a terrain by specifying the shapes and sizes of obstacles. The effect of obstacles on signal propagation is determined by a static table based on the type of obstacle. [20] presented the principles of a WCDMA radio network simulator that accounts for path-loss, shadowing, and fast fading effects in radio signal propagation. In contrast, we propose an empirically derived paramerizable model for the effect of obstacles on radio propagation.

\section{CONCLUSIONS}

We investigated two mesh-enhanced VANET deployment scenarios: (1) a mesh-enhanced peer-to-peer ad hoc routing (MEPPR) deployment in which mesh nodes as well as mobile nodes relay packets in the network, (2) a mesh-enhanced infrastructural routing (MEIR) deployment in which only the mesh nodes route packets. We examined impact of factors such as availability of multiple radio channels for communication and radio signal attenuation due to physical obstacles using a mobility model in which vehicular movement is constrained by traffic rules and street layout. Our results show that MEIR improves performance in dense networks by reducing the churn in network connectivity. On the other hand, MEPPR improves performance in sparse networks by exploiting opportunistic connectivity among mobile nodes. We also show that the impact of obstacles on radio propagation could be modeled in simulations through a few tunable parameters derived from empirical measurements.

\section{REFERENCES}

[1] D. Johnson, D. Maltz, and J. Broch, The Dynamic Source Routing Protocol for Multihop Wireless Ad Hoc Networks. Addison-Wesley, 2001, ch. 5, pp. 139-172.

[2] A. Mahajan, N. Potnis, K. Gopalan, and A. Wang, "Urban mobility models for VANETs," in Proc. of 2nd Workshop on Next Generation Wireless Networks, 2006.

[3] Wavemon: Wireless Device Monitoring Application, "http://packages.debian.org/unstable/net/wavemon."

[4] R. Jain, The Art of Computer Systems Performance Analysis. John Wiley and Sons Inc. New York, 1991.

[5] "Enhanced Network Simulator," http://www.cse.iitk.ac.in/users/braman/tens/.

[6] NS2 Network Simulator, "http://www.isi.edu/nsnam/ns/."

[7] A. Saha and D. Johnson, "Modeling mobility for vehicular ad-hoc networks," in Proc. of VANET, 2004.

[8] D. R. Choffnes and F. E. Bustamante, "An integrated mobility and traffic model for vehicular wireless networks," in VANET '05: Proc. of the 2nd ACM Intl. workshop on Vehicular ad hoc networks. New York, NY, USA: ACM Press, 2005, pp. 69-78.

[9] H. Wu, R. Fujimoto, R. Guensler, and M. Hunter, "Mddv: A mobility-centric data dissemination algorithm for vehicular networks," in VANET'04.

[10] M. Bechler, S. Jaap, and L. Wolf, "An optimized tcp for internet access of vehicular ad hoc networks," in Proc. of IFIP Networking, 2005.

[11] T. Little and A. Agarwal, "A new information propagation scheme for vehicular networks," in Proc. of Mobisys, 2005.

[12] H. Hartenstein, B. Bochow, A. Ebner, M. Lott, M. Radimirsch, and D. Vollmer, "Position-aware ad hoc wireless networks for inter-vehicle communications: The fleetnet project." in Proc of MobiHoc, 2001.

[13] D. Ghosal, C. Chuah, and M. Zhang, "Distributed sensing and computing with vehicular ad hoc networks," Computer Engineering Research Laboratory (CERL), University of California, Davis, Tech. Rep. ECECE-2004-9, 2004

[14] Self-Organizing Neighborhood Wireless Mesh Networks, "http://research.microsoft.com/mesh/."

[15] Roofnet, "http://pdos.csail.mit.edu/roofnet/doku.php."

[16] Mesh-Enabled Solutions for Intelligent Transportation, "http://www.motorola.com/mesh/pages/applications/its.htm."

[17] A. Raniwala, K. Gopalan, and T. Chiueh, "Centralized algorithms for multi-channel wireless mesh networks," 2004.

[18] A. Cavilla, G. Baron, T. Hart, L. Litty, and E. de Lara, "Simplified simulation models for indoor MANET evaluation are not robust," in Proc. of the IEEE SECON, Oct. 2004.

[19] A. Jardosh, E. Belding-Royer, K. Almeroth, and S. Suri, "Towards realistic mobility models for mobile ad hoc networks," in Proc. of MobiCom, 2003.

[20] S. Hamalaninen, H. Holma, and K. Sipil, "Advanced WCDMA Radio Network Simulator," in Proc. of the IEEE Intl. Symposium on Personal, Indoor and Mobile Radio, Osaka, Japan, Sep. 1999. 